\title{
Accelerated Alcoholic Fermentation of Intact Grapes by Saccharomyces Cerevisiae in Symbiosis with Microbial Community Inhabiting Grape-skin
}

Daisuke Watanabe

Kyoto University https://orcid.org/0000-0002-8831-5765

Wataru Hashimoto ( $\square$ hashimoto.wataru.8c@kyoto-u.ac.jp)

Kyoto University

\section{Article}

Keywords: Accelerated Alcoholic Fermentation, Intact Grapes, Saccharomyces Cerevisiae, Symbiosis

Posted Date: September 23rd, 2021

DOl: https://doi.org/10.21203/rs.3.rs-916454/v1

License: (c) (1) This work is licensed under a Creative Commons Attribution 4.0 International License.

Read Full License 
2 Accelerated alcoholic fermentation of intact grapes by Saccharomyces 3 cerevisiae in symbiosis with microbial community inhabiting grape-skin

5 Daisuke Watanabe $^{1} \&$ Wataru Hashimoto ${ }^{1 *}$

6

$7 \quad{ }^{1}$ Laboratory of Basic and Applied Molecular Biotechnology, Division of Food Science 8 and Biotechnology, Graduate School of Agriculture, Kyoto University, Uji, Kyoto, Japan 9

$10 \quad$ *email: hashimoto.wataru.8c@kyoto-u.ac.jp 


\section{Abstract}

2 Saccharomyces cerevisiae, an essential player in alcoholic fermentation during 3 winemaking, is rarely found in intact grapes. Here, we addressed symbiotic interactions

4 between $S$. cerevisiae and grape-skin residents upon spontaneous wine fermentation.

5 When glucose was used as a carbon source, the yeast-like fungus Aureobasidium

6 pullulans, a major grape-skin resident, had no effect on alcoholic fermentation by $S$.

7 cerevisiae. In contrast, when intact grape berries as a sole carbon source, coculture of $S$.

8 cerevisiae and A. pullulans accelerated alcoholic fermentation. Thus, grape-inhabiting

9 microorganisms may increase carbon availability by degrading and/or incorporating 10 grape-skin materials, such as cell wall and cuticles. A. pullulans exhibited broad spectrum 11 assimilation of plant-derived carbon sources, including $\omega$-hydroxy fatty acids, arising

12 from degradation of cutin. In fact, yeast-type cutinase was produced from A. pullulans

13 EXF-150 strain. The degradation and utilization of grape-skin materials by fungal

14 microbiota may account for their colonization on grape-skin and symbiotic interactions

15 with S. cerevisiae. 


\section{Introduction}

2 The origin of wine yeasts, taxonomically categorized into Saccharomyces cerevisiae or

3 its closely related species, has been an issue of great controversy. Historically, wine yeasts

4 were first found on grape surfaces ${ }^{1}$, whereas Saccharomyces species are absent or rare in

5 fresh grape berries ${ }^{2-4}$. Resident microbiota in intact grapes predominantly consists of the

6 yeast-like ascomycetous fungi Aureobasidium pullulans and basidiomycetous yeasts,

7 such as Cryptococcus, Rhodosporidium, Rhodotorula, and Sporobolomyces (a.k.a.

8 Sporidiobolus as teleomorphic forms) species, which are irrelevant to winemaking due to

9 their lack of alcoholic fermentation ability ${ }^{3-5}$. Assuming that Saccharomyces yeast species

10 are vectored by insects or migratory birds $s^{6,7}$, the first colonist wine yeasts must endure

11 and adapt in vineyard environments, nutritionally undesirable for survival and

12 proliferation.

The grape-skin, the natural habitat for non-Saccharomyces oligotrophic microorganisms, covers and protects the pulp for nutrient storage ${ }^{8,9}$. The grape-skin occupies approximately $10 \%$ of the dry weight of grape berries and acts as a barrier against dehydration, physical damage, and microbial penetration. The plant primary cell wall components, cellulose, hemicellulose, and pectin, contribute to the structural integrity. The grape-skin is particularly abundant in pectinic acid, the methyl-esterified

19 form of polygalacturonic acid ${ }^{10,11}$. As previously reported, many grape-inhabiting 20 microorganisms secrete cellulase, pectinase, and relevant degrading enzymes to use the 21 cell wall decomposition products as nutrients ${ }^{12-15}$. The microbial targeting ability to degrade and assimilate the plant cell wall compounds is likely a prerequisite for colonization and adaptation to vineyard environments. Wine yeasts of the Saccharomyces genus may need the aid of grape-skin residents to survive on the surface of grapes due to 
1 the absence of degrading or metabolizing enzymes for plant cell wall and decomposition 2 products $^{16,17}$.

The outermost layer of grape-skin is the cuticle, mainly composed of the lipid polyester cutin (i.e., ester-linked $\omega$-hydroxy $\mathrm{C}_{16}$ and $\mathrm{C}_{18}$ fatty acids) and the chemically highly resistant biopolymer cutan ${ }^{18,19}$. On all aerial surfaces of land plants, thick and hydrophobic cuticle layers prevent desiccation, UV damage, and pathogen infection. At the frontline, the plant cuticle also serves multifunctional roles in triggering immunity during plant-pathogen interactions ${ }^{20}$. Thus, cuticle-degrading virulent pathogens can cause severe damage to terrestrial plants. The cutin-hydrolyzing enzyme cutinase has been extensively studied in typical plant pathogens of Fusarium species and in a limited number of molds, yeasts, and bacteria ${ }^{21-23}$. However, the degradation and assimilation of the plant cuticle compounds by non-pathogenic, grape-skin microorganisms are yet to be described.

This study proposed a novel tripartite relationship between grape berries, grapeskin microbiota, and S. cerevisiae during alcoholic fermentation. Similar plant-microbial interactions may typically occur in traditional production processes of fermented foods and beverages, where humans have optimized the growth of characteristic microbial communities over thousands of years ${ }^{24}$. Thus, this study provides an important clue to understand the dynamics and mechanism of plant-microbial ecosystems by analyzing the origin of wine fermentation as an experimentally tractable model.

\section{Results}

Isolation of yeasts and related microorganisms inhabiting grape-skin. Among chloramphenicol-tolerant, yeast-like colonies isolated from grape juice, surface-washed 
1 suspensions, or enrichment cultures, 76 clones were identified to species level by 2 sequencing the internal transcribed spacer (ITS) region of the nuclear rRNA gene 3 (Supplementary Table S1). Isolated microorganisms were classified as yeast-like fungi, 4 basidiomycetous yeasts, or ascomycetous yeasts. The yeast-like fungi included a single 5 ascomycetous species $A$. pullulans ${ }^{25-27}$, also known as "black yeast", frequently isolated 6 from grape and wine environments in previous reports ${ }^{3-5}$. On nutrient-rich yeast extract7 peptone-dextrose (YPD) medium, A. pullulans earlier produced smooth pale-pink 8 colonies, which developed protrusions after long-term cultivation (Fig. 1a). Due to 9 melanin-like pigment accumulation, A. pullulans cells were easily observed on the skin 10 of spoiled grape berries (Fig. 1b). The isolated basidiomycetous yeasts mainly consisted of Sporobolomyces and Papiliotrema (formerly categorized as Cryptococcus) species $^{3-5}$. Additionally, Rhodotorula mucilaginosa is a common ecological basidiomycete found in soil, air, water, and foods ${ }^{26,28}$. Hanseniaspora uvarum ${ }^{26,29}$ was most frequently isolated 14 from the wine grape variety, Pinot noir, among the ascomycetous yeast species. Several other ascomycetes were assigned to Candida (as anamorphic status) or Pichia genus. In most cases, every grape sample tested in this study contained a few yeasts or associated fungal species, excluding Saccharomyces cerevisiae. Thus, grape environments may be greatly suitable for the colonization and development of non-Saccharomyces oligotrophic fungal microbiota. Yeast-like fungus A. pullulans, three basidiomycetous yeast species (Papiliotrema laurentii, Sporidiobolus pararoseus, and $R$. mucilaginosa), and five ascomycetous yeast species (H. uvarum, Torulaspora delbrueckii, Meyerozyma caribbica, Debaryomyces hansenii, and Pichia terricola) were used as representative grape-skin microorganisms for further comparative analysis (Fig. 2). 
1 Alcoholic fermentation of glucose by grape-skin fungi and $S$. cerevisiae. To assess

2 alcoholic fermentation performance of the grape-skin fungi and $S$. cerevisiae, each 3 microorganism was statically incubated in a 5-mL liquid yeast nitrogen base (YNB) 4 medium, synthetic minimum medium for laboratory yeast strains, supplemented with $510 \%(\mathrm{w} / \mathrm{v})$ glucose as a sole carbon source. As all species grew well in this medium 6 (Supplementary Fig. S1; optical density of $600 \mathrm{~nm}\left(\mathrm{OD}_{600}\right)>1$ ), carbon dioxide emission

7 rate, glucose consumption, and ethanol production were quantified during the 6-d 8 fermentation test (Fig. 3). S. cerevisiae exhibited a robust peak of fermentation rate and 9 full glucose consumption to yield approximately 5\% (v/v) ethanol. In contrast, yeast-like fungus A. pullulans, basidiomycetous yeasts, $P$. laurentii, S. pararoseus, $R$. mucilaginosa, and ascomycetous yeast $D$. hansenii showed a constant basal level of carbon dioxide emission, no detectable ethanol production, and little glucose consumption, and was categorized as non-fermenting species. The other ascomycetous yeasts, $H$. uvarum, $T$. glucose as a sole carbon source, the non-fermenting species A. pullulans displayed no significant interaction with S. cerevisiae in alcoholic fermentation (Fig. 4a). Notably, the coculture of A. pullulans and S. cerevisiae had no synergistic effect on the growth of both species (Supplementary Fig. S2).

Alcoholic fermentation of intact grapes by nonfermentative, grape-skin fungi and $S$. cerevisiae. In 50-mL YNB minimum medium plus 50-g intact grape berries as a sole carbon source (Fig. 4b), A. pullulans generated almost no carbon dioxide, whereas $S$. 
1 cerevisiae slowly progressed alcoholic fermentation, reaching the maximum carbon

2 emission rate $4 \mathrm{~d}$ after inoculation. Since robust peaks of fermentation rates were typically 3 observed within 1-2 d after inoculation in the presence of glucose (Figs. 3a and 4a), 4 grape-skin may function as a physical barrier against $S$. cerevisiae cells to protect 5 fermentable sugars, such as glucose, fructose, and sucrose, inside grape berries. When A. pullulans and S. cerevisiae were co-cultured, the initial carbon emission

7 rate increased, and a maximum fermentation rate was observed at $3 \mathrm{~d}$ (Fig. 4b).

8 Considering the minor contribution of A. pullulans to alcoholic fermentation and $S$. 9 cerevisiae growth, A. pullulans may specifically contribute to an increase in sugar availability for S. cerevisiae, leading to accelerated alcoholic fermentation. Coculture of S. cerevisiae with the nonfermentative basidiomycetous yeast, $P$. laurentii, S. pararoseus, or R. mucilaginosa, gave similar results (Supplementary Fig. S3). These data suggest that S. cerevisiae cells make use of the ability of grape-skin microbiota to access fermentable sugars in grape berries upon triggering spontaneous wine fermentation.

Carbon assimilation profiles of grape-skin fungi and $S$. cerevisiae. To characterize carbon assimilation profile, A. pullulans and S. cerevisiae cells were cultivated in a YNB minimum medium with various possible carbon sources in grape berries (Fig. 5). Both $A$. pullulans and S. cerevisiae vigorously grew in the presence of fermentable sugars, such as glucose and sucrose (Fig. 5a, b). A major part of grape-skin consists of plant cell wall polymers, cellulose and pectin ${ }^{10}$. Although carboxymethyl cellulose (CMC), a watersoluble cellulose derivative, was used by neither A. pullulans nor S. cerevisiae, the main cellulose degradation product cellobiose was assimilated by A. pullulans (Fig. 5c, d). As previously reported ${ }^{30}$, the activity of carboxymethyl cellulase (i.e., CMCase) is deficient 
1 among some A. pullulans strains. The A. pullulans cells identified in this study may be

2 unable to degrade cellulose by themselves but can grow by using cellobiose produced 3 through cellulose degradation by other grape-skin fungi. The major pectic polysaccharide 4 polygalacturonic acid and its building block galacturonic acid were assimilated by $A$. 5 pullulans, but not by $S$. cerevisiae (Fig. 5e, f). Thus, A. pullulans likely degrades and 6 assimilates a broad spectrum of plant cell wall-relevant materials, unmetabolized by $S$. 7 cerevisiae, consistent with previous reports ${ }^{12-15,26,27}$.

The plant cuticle, the outermost hydrophobic layer, is another major component 9 of grape-skin ${ }^{10}$. Cutin in the plant cuticle is a polyester of $\omega$-hydroxy $\mathrm{C}_{16}$ and $\mathrm{C}_{18}$ fatty 10 acids and their derivatives. We discovered that $A$. pullulans exhibited weak but reproducible growth using $\omega$-hydroxypalmitic acid as a sole carbon source (Fig. 5g). Since neither palmitic acid or $\omega$-carboxypalmitic acid was assimilated (Fig. 5h, i), A. pullulans may possess a utilization system specific for $\omega$-hydroxy fatty acids. Besides $A$. pullulans, three basidiomycetous yeasts, $P$. laurentii, S. pararoseus, and $R$. mucilaginosa, and two ascomycetous yeasts, $M$. caribbica and $D$. hansenii, showed significant growth in the presence of $\omega$-hydroxy palmitic acid (Fig. 6). Notably, most species described above were nonfermentative microorganisms.

Cutinase-like esterase activity in A. pullulans. The secretion of cutin-degrading enzymes in A. pullulans, basidiomycetous yeasts, and $S$. cerevisiae was tested using a model polyester polycaprolactone (PCL)-plate (Fig. 7a). Known cutinases from the other species represent PCL degradation activity in previous studies ${ }^{31-33}$. The supernatant of the fully grown A. pullulans culture in YNB medium plus $2 \%$ glucose formed a clear halo, although no halo was observed using the other supernatant samples. Additionally, the 
1 supernatant of the A. pullulans culture exhibited higher esterase activity toward $p$ 2 nitrophenyl butyrate $(p N P B)$ and $p$-nitrophenyl palmitate ( $p$ NPP) used as substrates than 3 the supernatant of the $S$. cerevisiae culture (Fig. 7b). These data suggest that the isolated 4 A. pullulans strain from grapes secretes cutinase to assist penetration into plant cuticles.

Previous whole-genome analysis of the A. pullulans EXF-150 strain ${ }^{34}$ revealed nine candidate genes encoding cutinase-like enzymes, designated as ApCut1 to ApCut9 (Supplementary Fig. S4). These gene products contained a classical $\alpha / \beta$-hydrolase catalytic triad Ser-His-Asp and a Gly-Tyr-Ser-Gln-Gly (GYSQG) motif conserved among cutinase catalytic sites ${ }^{22,27}$. Additionally, two pairs of cysteine residues forming disulfide bonds, important for spatial conformation ${ }^{22,35}$, were also conserved in all cutinase candidates except for ApCut4, in which the amino terminus is truncated. Phylogenetic analysis indicated that ApCut1 to ApCut3 form a subgroup with yeast cutinases from $A$. adeninivorans and Cryptococcus sp. S-2 $2^{31,33}$, while ApCut5 to ApCut9 form a subgroup with mold cutinases from F. solani, A. oryzae, and B. cinerea (Fig. 8a) ${ }^{36-38}$. Whole-cell lysate of E. coli expressing recombinant ApCut1 indicated cutinase-like activity, based on the PCL-plate clearing assay and the $p$ NPB hydrolysis assay (Fig. 8b-d). Also, the extracts of ApCut2- or ApCut3-expressing E. coli cells weakly degraded PCL (Supplementary Fig. S5). Since the expression of ApCut2 and ApCut3 was almost undetectable in the coomassie brilliant blue (CBB)-stained gel, more attention should be paid to the protease sensitivity, expression conditions, and synonymous codon usage bias. These results revealed the activity of the yeast-type cutinase isoenzymes in A. pullulans. 


\section{Discussion}

2 Based on the experimental data, we propose that the grape-skin-resident microorganisms,

3 including nonfermentative, yeast-like fungus A. pullulans, increase the accessibility to

4 fermentable sugars in intact grape berries by degrading and assimilating the plant cell

5 wall, cuticle compounds, or both. Grape-skin microbiota's high and versatile abilities to

6 degrade and assimilate plant cell wall and/or cuticle is likely to be essential for their

7 adaptation and proliferation on grape-skin. In contrast, S. cerevisiae cells without such

8 abilities need the aid of grape-skin microbiota to survive in grape environments. Studies

9 of wild populations of S. cerevisiae and its closest relative Saccharomyces paradoxus

10 suggest that woodlands or primeval forests are natural habitats for these yeast species ${ }^{39-}$

$11{ }^{41}$. In this study, no S. cerevisiae clone was isolated even from fermented juice or enriched

12 cultures in 5\% sucrose. Moreover, S. cerevisiae was unable to assimilate plant cell wall,

13 cuticle, and their components. Altogether, nutrient-poor, intact grape surfaces may be

14 inappropriate for wine yeasts as their stable and permanent habitats. Although

15 Saccharomyces species might have been accidentally brought to the vineyard by yeastcarrier animals ${ }^{6,7}$, the primary cause of wine yeasts still needs to be experimentally explored. Our results provide an important clue to address how S. cerevisiae cells met and conquered grapes upon the origin of spontaneous wine fermentation.

What are the key grape-skin compounds that protect fermentable sugars in intact grape berries? Based on the assimilation tests of CMC and cellobiose (Fig. 5c, d), our isolated A. pullulans strain lacks cellulase. This is consistent with a previous report about intraspecific variations of cell wall-degrading enzymes in A. pullulans ${ }^{30}$. Thus, cellulose degradation may be nonessential for accelerated alcoholic fermentation of intact grapes.

24 Among pectin-degrading enzymes, polygalacturonase is genetically encoded and 
1 expressed by grape-skin resident species and by S. cerevisiae $e^{42,43}$. Recently, we revealed

2 the importance of pectin as an initial target for the saprophytic bacterium Bacillus subtilis

3 to recognize the surface of dead soybeans ${ }^{44}$, whereas it is unlikely that pectin degradation

4 by non-Saccharomyces microorganisms specifically accelerated alcoholic fermentation

5 of intact grapes. As shown in Fig. 7a, the PCL-degrading activity was detected in $A$.

6 pullulans, although not in the examined basidiomycetous yeast strains. Based on these

7 data, the responsible enzymatic activity specific and common to all grape-skin residents

8 is still unidentified. The degradation of the other plant cell wall or cuticle components

9 should be focused on in future research. Alternatively, cooperative or synergistic

10 degradation of plant epidermis by whole grape-skin microbiota needs to be investigated.

11 Such complicated, highly ordered microbial interactions at the chemical, metabolic,

12 genetic, and genomic levels will be the central issue in applied microbiology and

13 microbial ecology.

14 This is the first report on $\omega$-hydroxypalmitic acid assimilation as a carbon source

by $A$. pullulans and several yeast species. Although $\omega$-hydroxylation of fatty acids also occurs in mammals and insects, $\omega$-hydroxy fatty acids play a broad and vital biological role in higher plants as major components of cutin and suberin ${ }^{18-20,45-47}$. Thus, the microbial ability to assimilate $\omega$-hydroxy fatty acids may mainly contribute to symbiotic interactions with terrestrial plants. In the $\omega$-oxidation process of animals and plants, known as a minor, fatty acid catabolic pathway, a hydroxy group is introduced onto the $\omega$ carbon of the medium to long-chain fatty acids ${ }^{46,47}$. The resultant $\omega$-hydroxy fatty acids are oxidized to $\omega$-carboxy fatty acids (i.e., dicarboxylic fatty acids), further degraded through the $\beta$-oxidation pathway. Grape-skin residents may probably metabolize $\omega$ hydroxy fatty acids similarly. Based on our data, A. pullulans cells are suggested to have 
1 an $\omega$-hydroxy fatty acid-specific transporter because they can grow using $\omega$ 2 hydroxypalmitic acid as a sole carbon source not palmitic acid or $\omega$-carboxypalmitic acid.

3 Furthermore, this study revealed that A. pullulans may secrete cutinase to hydrolyze cutin 4 into $\omega$-hydroxy fatty acids and other minor components, such as glycerol. The 5 combination of cutin degradation and $\omega$-hydroxy fatty acid assimilation may characterize

6 A. pullulans as the most abundant and persistent resident among grape-skin microbiota.

7 Notably, the A. pullulans EXF-150 strain was first identified as a microorganism that 8 acquired both yeast- and mold-type cutinase genes in the genome, which may be 9 associated with the yeast-to-hyphal dimorphic transition of this species ${ }^{25}$. Enzymatic 10 analysis of yeast- and mold-type cutinases in A. pullulans will reveal the significance of differences between both cutinase types.

Conclusively, this study focused on the symbiotic relationship between grapeskin microbiota and $S$. cerevisiae from the perspective of winemaking origin. 14 Oligotrophic microorganisms, such as A. pullulans, have developed versatile abilities to use plant cell wall polysaccharides and plant cuticular lipids as nutrient sources to establish their ecological niche. Especially, degradation and assimilation of the plant cuticle, the outermost layer interacting with the environment, may be a prerequisite for oligotrophic resident microorganisms to trigger colonization and adaptation. In contrast, eutrophic yeasts, including S. cerevisiae, yield energy through the alcoholic fermentation of sugars in grape berries with the aid of oligotrophic microorganisms. Such tripartite interaction between grape berries, oligotrophic residents, and eutrophic yeasts determines the grape-skin microbiome dynamics and spontaneous wine fermentation. Thus, studying the origins and microbiota ecological succession in fermented foods will help elucidate the key principles governing plant-microbial ecosystems' emergence and development. 


\section{Materials}

Materials and strains. To isolate yeasts or yeast-like fungi, (i) juice, (ii) surface-washed suspensions, or (iii) enrichment cultures were obtained from commercially available wine or table grape varieties belonging to Vitis vinifera species or Vitis interspecific hybrids (see Supplementary Table S1). Grape juice was obtained from freshly pressed grape berries using a food-grade juicer (Panasonic, Japan) and was used either immediately or after being incubated at $30^{\circ} \mathrm{C}$ for $3 \mathrm{~d}$. Surface-washed suspensions were obtained by vigorously shaking the flask containing approximately 50 -g grape berries and $25-\mathrm{mL}$ sterilized water at $30^{\circ} \mathrm{C}$ for $15 \mathrm{~min}$. Enrichment cultures were obtained by statically incubating the flask containing approximately 10-g grape berries and 40-mL sterilized water or $5 \%(\mathrm{w} / \mathrm{v})$ sucrose at $30^{\circ} \mathrm{C}$ for $3 \mathrm{~d}$. Each sample was spread on a nutrientrich,YPD ( $1 \%$ yeast extract, $2 \%$ peptone, and $2 \%$ glucose) medium plate with $0.1 \%$ chloramphenicol to inhibit bacterial growth, and was incubated at $30^{\circ} \mathrm{C}$. Single colonies representing yeast-like colony morphology were isolated by repeatedly streaking on YPD medium plates with $0.1 \%$ chloramphenicol. The isolated clones were identified by DNA sequencing of the rRNA gene ITS region, using the ITS_1F (5'GTAACAAGGTYTCCGT-3') and ITS_1R (5'-CGTTCTTCATCGATG-3') primer pair and genomic DNA as PCR templates. The $S$. cerevisiae X2180 strain, obtained from the American Type Culture Collection (USA), was used for alcoholic fermentation tests or other control experiments.

Fermentation test. Cells were aerobically precultured at $30^{\circ} \mathrm{C}$ for $2 \mathrm{~d}$ in $0.67 \%$ YNB minimum medium containing $2 \%(\mathrm{w} / \mathrm{v})$ glucose as a carbon source and harvested. For the alcoholic fermentation of glucose, cells were inoculated into a YNB medium containing 
$110 \%(\mathrm{w} / \mathrm{v})$ glucose at a final $\mathrm{OD}_{600}$ of 0.1 and were then further incubated at $30^{\circ} \mathrm{C}$ without

2 shaking. For the alcoholic fermentation of intact grapes, cells were inoculated into 50-mL

3 YNB medium at a final $\mathrm{OD}_{600}$ of 0.1 , mixed with approximately 50 -g commercially

4 available intact grape berries of Green Seedless, and were then further incubated at $30^{\circ} \mathrm{C}$

5 without shaking. Fermentation was continuously monitored by measuring the weight loss

6 of evolved carbon dioxide for 5-mL test tube-scale tests or using a Fermograph II

7 apparatus (Atto, Japan) for 50-mL-scale coculture tests. Glucose and ethanol

8 concentrations were determined using the LabAssay glucose kit (Fujifilm Wako Pure

9 Chemical, Japan) and the ethanol assay F-kit (Roche, Switzerland), respectively.

11 Carbon assimilation test. Cells were aerobically precultured at $30^{\circ} \mathrm{C}$ for $2 \mathrm{~d}$ in a YNB minimum medium containing $2 \%(\mathrm{w} / \mathrm{v})$ glucose as a carbon source, harvested, washed by sterilized water, and inoculated into a YNB medium containing $0.5 \%(\mathrm{w} / \mathrm{v})$ of carbon sources as below: glucose, sucrose, $\mathrm{CMC}$, cellobiose, polygalacturonic acid, galacturonic acid, palmitic acid, 16-hydroxyhexadecanoic acid (i.e., $\omega$-hydroxypalmitic acid), or heptadecanedioic acid (i.e., $\omega$-carboxypalmitic acid). Upon inoculation, initial $\mathrm{OD}_{600}$ was adjusted to 0.1 . For assimilation tests of palmitic acid, $\omega$-hydroxypalmitic acid, or $\omega$ carboxypalmitic acid, $0.05 \%$ (w/v) Tween 40 was added to the medium. In advance of measurement of $\mathrm{OD}_{600}$, insoluble fatty acids were removed by washing pellets with hexane three times, and cells were dissolved in sterilized water ${ }^{48}$.

Phylogenetic analysis of yeast and mold cutinases. Amino acid sequences of nine putative cutinase gene products in the A. pullulans EXF-150 strain and representative yeast and mold cutinases [Cut2 from Arxula adeninivorans (AaCut2), Cle1 from 
$1 \quad$ Cryptococcus sp. strain S-2 (CS2Cle1), Cut1 from Fusarium solani (FsCut1), CutL from

2 Aspergillus oryzae (AoCutL), and CutA from Botrytis cinerea (BcCutA)] were obtained

3 from UniPlotKB. Multiple sequence alignment was conducted using Clustal Omega

4 program, and the phylogenetic tree was constructed using Molecular Evolutionary

5 Genetics Analysis v.10.2.2. The putative cutinase genes of $A$. pullulans were designated

6 as below: M438DRAFT_340638 as ApCUT1, M438DRAFT_351543 as ApCUT2,

7 M438DRAFT_352218 as ApCUT3, M438DRAFT_388226 as ApCUT4,

8 M438DRAFT_264999 as ApCUT5, M438DRAFT 267580 as ApCUT6,

9 M438DRAFT_341517 as ApCUT7, M438DRAFT_347465 as ApCUT8, and

10 M438DRAFT_368700 as ApCUT9.

Expression of recombinant $A$. pullulans cutinase. The chemically synthesized ApCUT1 gene (Eurofins, Luxembourg) was cloned into the BamHI-EcoRI site of the pET-21b(+) vector (Merck Millipore, USA) to express recombinant ApCut1p tagged with the T7 epitope at the amino terminus and the $6 \times$ His epitope at the carboxy terminus. The resultant pET-21b(+)-ApCUT1 plasmid was introduced into Escherichia coli BL21(DE3)pLysS cells (Novagen, Germany). A transformant was inoculated into 100mL Luria-Bertani medium ( $0.5 \%$ yeast extract, $1 \%$ tryptone, and $1 \%$ sodium chloride) with $100-\mu \mathrm{g} / \mathrm{mL}$ ampicillin and $34-\mu \mathrm{g} / \mathrm{mL}$ chloramphenicol, and was cultured at $37^{\circ} \mathrm{C}$ to an $\mathrm{OD}_{600}$ of 0.6 . Isopropyl thio- $\beta$-D-galactoside (IPTG) was added to the culture to a final concentration of $1 \mathrm{mM}$, and cells were further cultured at $37^{\circ} \mathrm{C}$ for $4 \mathrm{~h}$. Whole-cell extracts were prepared from cell pellets suspended in the xTractor buffer (TakaraBio, Japan), followed by the addition of DNase I. After protein separation by SDS-PAGE, the proteins were detected by CBB staining or using His-Detect In-Gel Stain (Nacalai Tesque, 
1 Japan).

2

3 Enzymatic assay. To assay the potential cutinase activity from the culture supernatants

4 of fully grown A. pullulans or yeasts, PCL was used as a model polyester substrate. Turbid 5 agar plates containing $0.05 \%$ PCL (Fujifilm Wako Pure Chemical, Japan) were prepared 6 as previously described ${ }^{32,33}$. The assay for determining esterase activity was conducted 7 according to previous reports ${ }^{33,49}$, using $p$ NPB and $p$ NPP (Sigma-Aldrich, USA) as 8 substrates. 


\section{References}

2 1. Pasteur L. Nouvelles expériences pour démontrer que le germe de la levure qui fait le vin provient de l'extérieur des grains de raisin. C R Acad Sci 1872; 75: 781-793.

2. Mortimer R, Polsinelli M. On the origins of wine yeast. Res Microbiol 1999; 150: 199-204.

3. Barata A, Malfeito-Ferreira M, Loureiro V. The microbial ecology of wine grape berries. Int J Food Microbiol 2012; 153: 243-259.

4. Stefanini I, Cavalieri D. Metagenomic approaches to investigate the contribution of

9. Martínez-Lapuente L, Guadalupe Z, Ayestarán B. Properties of wine polysaccharides. 
In: Masuelli M (ed). Pectins - Extraction, Purification, Characterization and Applications. IntechOpen: 2019, DOI: 10.5772/intechopen.85629.

10. Lecas M, Brillouet J-M. Cell wall composition of grape berry skins. Phytochemistry 1994; 35: 1241-1243.

11. González-Centeno MR, Rosselló C, Simala S, Garau MC, López F, Femenia A. Physico-chemical properties of cell wall materials obtained from ten grape varieties and their byproducts: grape pomaces and stems. LWT Food Sci Technol 2010; 43: $1580-1586$.

12. Biely P, Heinrichová K, Kružiková M. Induction and inducers of the pectolytic system in Aureobasidium pullulans. Curr Microbiol 1996; 33: 6-10.

13. Strauss ML, Jolly NP, Lambrechts MG, van Rensburg P. Screening for the production of extracellular hydrolytic enzymes by non-Saccharomyces wine yeasts. J Appl Microbiol 2001; 91: 182-190.

14. Merín MG, Mendoza LM, Farías ME, Morata de Ambrosini VI. Isolation and selection of yeasts from wine grape ecosystem secreting cold-active pectinolytic activity. Int J Food Microbiol 2011; 147: 144-148.

15. Úbeda J, Maldonado Gil M, Chiva R, Guillamón JM, Briones A. Biodiversity of nonSaccharomyces yeasts in distilleries of the La Mancha region (Spain). FEMS Yeast Res 2014; 14: 663-673.

16. Huisjes EH, Luttik MAH, Almering MJH, Bisschops MMM, Dang DHN, Kleerebezem M et al. Toward pectin fermentation by Saccharomyces cerevisiae: expression of the first two steps of a bacterial pathway for D-galacturonate metabolism. J Biotechnol 2012; 162: 303-331.

17. Casa-Villegas M, Polaina J, Marín-Navarro, J. Cellobiose fermentation by 
Saccharomyces cerevisiae: comparative analysis of intra versus extracellular sugar hydrolysis. Process Biochem 2018; 75: 59-67.

18. Domínguez E, Heredia-Guerrero JA, Heredia A. The biophysical design of plant cuticles: an overview. New Phytol 2011; 189: 938-949.

19. Martin LBB, Rose JKC. There's more than one way to skin a fruit: formation and functions of fruit cuticles. J Exp Bot 2014; 65: 4639-4651.

20. Ziv C, Zhao Z, Gao YG, Xia Y. Multifunctional roles of plant cuticle during plantpathogen interactions. Front Plant Sci 2018; 9: 1088.

21. Egmond MR, de Vlieg J. Fusarium solani pisi cutinase. Biochimie 2000; 82: 10151021.

22. Chen S, Su L, Chen J, Wu J. Cutinase: characteristics, preparation, and application. Biotechnol Adv 2013; 31: 1754-1767.

23. Nyyssölä A. Which properties of cutinases are important for applications? Appl Microbiol Biotechnol 2015; 99: 4931-4942.

24. Wolfe BE, Dutton RJ. Fermented foods as experimentally tractable microbial ecosystems. Cell 2015; 161: 49-55.

25. Slepecky RA, Starmer WT. Phenotypic plasticity in fungi: a review with observations on Aureobasidium pullulans. Mycologia 2009; 101: 823-832.

26. Varela C, Borneman AR. Yeasts found in vineyards and wineries. Yeast 2017; 34: $111-128$.

27. Bozoudi D, Tsaltas D. The multiple and versatile roles of Aureobasidium pullulans in the vitivinicultural sector. Fermentation 2018; 4: 85 .

28. Wirth F, Goldani LZ. Epidemiology of Rhodotorula: an emerging pathogen. Interdiscip Perspect Infect Dis 2012; 2012: 465717. 
29. Martin V, Valera MJ, Medina K, Boido E, Carrau F. Oenological impact of the Hanseniaspora/Kloeckera yeast genus on wines--a review. Fermentation 2018; 4: 76.

30. Sun P-F, Chien I-A, Xiao H-S, Fang W-T, Hsu C-H, Chou J-Y et al. Intraspecific variation in plant growth-promoting traits of Aureobasidium pullulans. Chiang Mai J. Sci. 2019; 46: 15-31.

31. Masaki K, Kamini NR, Ikeda H, Iefuji H. Cutinase-like enzyme from the yeast Cryptococcus sp. strain S-2 hydrolyzes polylactic acid and other biodegradable plastics. Appl Environ Microbiol 2005; 71: 7548-7550.

32. Murphy CA, Cameron JA, Huang SJ Vinopal RT. Fusarium polycaprolactone depolymerase is cutinase. Appl Environ Microbiol 1996; 62: 456-460.

33. Bischoff F, Litwińska K, Cordes A. Three new cutinases from the yeast Arxula adeninivorans that are suitable for biotechnological applications. Appl Environ Microbiol 2015; 81: 5497-5510.

34. Gostinčar C, Ohm RA, Kogej T, Sonjak S, Turk M, Zajc J et al. Genome sequencing of four Aureobasidium pullulans varieties: biotechnological potential, stress tolerance, and description of new species. BMC Genomics 2014; 15: 549.

35. Kodama Y, Masaki K, Kondo H, Suzuki M, Tsuda S, Nagura T et al. Crystal structure and enhanced activity of a cutinase-like enzyme from Cryptococcus sp. strain S-2. Proteins 2009; 77: 710-717.

36. Soliday CL, Dickman MB, Kolattukudy PE. Structure of the cutinase gene and detection of promoter activity in the 5 '-flanking region by fungal transformation. $J$ Bacteriol 1989; 171: 1942-1951.

37. Ohnishi K, Toida J, Nakazawa H, Sekiguchi J. Genome structure and nucleotide sequence of a lipolytic enzyme gene of Aspergillus oryzae. FEMS Microbiol Lett 
1995; 126: 145-150.

38. van der Vlugt-Bergmans CJ, Wagemakers CA, van Kan JA. Cloning and expression of the cutinase A gene of Botrytis cinerea. Mol Plant Microb Interact 1997; 10: 2129.

39. Sniegowski PD, Dombrowski PG, Fingerman E. Saccharomyces cerevisiae and Saccharomyces paradoxus coexist in a natural woodland site in North America and display different levels of reproductive isolation from European conspecifics. FEMS Yeast Res 2002; 1: 299-306.

40. Fay JC, Benavides JA. Evidence for domesticated and wild populations of Saccharomyces cerevisiae. PLoS Genet 2005; 1: 66-71.

41. Duan S-F, Han P-J, Wang Q-M, Liu W-Q, Shi J-Y, Li K et al. The origin and adaptive evolution of domesticated populations of yeast from Far East Asia. Nat Commun 2018; 9: 2690.

42. Gognies S, Gainvors A, Aigle M, Belarbi A. Cloning, sequence analysis and overexpression of a Saccharomyces cerevisiae endopolygalacturonase-encoding gene (PGL1). Yeast 1999; 15: 11-22.

43. Radoi F, Kishida M, Kawasaki H. Endo-polygalacturonase in Saccharomyces wine yeasts: effect of carbon source on enzyme production. FEMS Yeast Res 2005; 5: 663668.

44. Sugiura H, Nagase A, Oiki S, Mikami B, Watanabe D, Hashimoto W. Bacterial inducible expression of plant cell wall-binding protein $\mathrm{YesO}$ through conflict between Glycine max and saprophytic Bacillus subtilis. Sci Rep 2020; 10: 18691.

45. Kolattukudy PE. Biopolyester membranes of plants: cutin and suberin. Science 1980; 208: 990-1000. 
1 46. Miura Y. The biological significance of $\omega$-oxidation of fatty acids. Proc Jpn Acad Ser B Phys Biol Sci 2013; 89: 370-382.

47. Wertz PW. Naturally occurring $\omega$-hydroxyacids. Int J Cosmet Sci 2018; 40: 31-33.

48. Nakagawa T, Imanaka T, Morita M, Ishiguro K, Yurimoto H, Yamashita A et al. Peroxisomal membrane protein Pmp47 is essential in the metabolism of middle-chain fatty acid in yeast peroxisomes and is associated with peroxisome proliferation. $J$ Biol Chem 2000; 275: 3455-3461.

49. Chen S, Tong X, Woodard RW, Du G, Wu J, Chen J. Identification and characterization of bacterial cutinase. J Biol Chem 2008; 283: 25854-25862.

\section{Acknowledgements}

This work was partly supported by Grants-in-Aid for Scientific Research from the Japan Society for the Promotion of Science (to D.W.). Addtionally, the authors want to thank Enago (https://www.enago.com) for the English language review.

Author contributions

7 W.H. designed the study. D.W. performed the experiments. D.W. and W.H. analyzed the data. D.W. and W.H. wrote the manuscript.

0 Competing interests

21 The authors declare no competing interests. 


\section{Figure legends}

2 Fig. 1 A. pullulans on grape-skin. a Colony morphology of an A. pullulans strain

3 isolated from Delaware grape berries in this study. Cells were streaked on YPD plate

4 medium with $0.1 \%$ chloramphenicol and were incubated at $37^{\circ} \mathrm{C}$ for $3 \mathrm{~d}$. Bar, $5 \mathrm{~mm}$. b $A$.

5 pullulans cells were observed on the surface of a spoiled Pinot noir grape berry. Arrows

6 indicate typical A. pullulans cells characterized by accumulation of melanin pigment. Bar

$7=10 \mu \mathrm{m}$.

9 Fig. 2 Representative grape-skin residents isolated in this study. Microscopic images

10 of the nine species of grape-skin residents and $S$. cerevisiae $(S c)$. Grape-skin residents include one yeast-like fungus $A$. pullulans $(A p)$, three basidiomycetous yeasts, $P$. laurentii

$(P l), S$. pararoseus $(S p)$, and $R$. mucilaginosa $(R m)$, and five ascomycetous yeasts, $H$. uvarum $(H u)$, T. delbrueckii $(T d)$, M. caribbica $(M c)$, D. hansenii $(D h)$, and P. terricola

$14(P t)$. Bars $=10 \mu \mathrm{m}$.

Fig. 3 Alcoholic fermentation of glucose by grape-skin residents and $S$. cerevisiae. a

17 Carbon dioxide emission rate during 6-d alcoholic fermentation in YNB medium containing $10 \%(\mathrm{w} / \mathrm{v})$ glucose. b Glucose consumption (orange) and ethanol production (yellow) during 6-d alcoholic fermentation in YNB medium containing 10\% (w/v) glucose. Data represent mean values and standard deviations from three independent experiments. Ap, A. pullulans; $P l, P$. laurentii; Sp, S. pararoseus; Rm, R. mucilaginosa; Hu, H. uvarum; Td, T. delbrueckii; Mc, M. caribbica; Dh, D. hansenii; Pt, P. terricola; Sc, S. cerevisiae. 
1 Fig. 4 Alcoholic fermentation of glucose or intact grapes in coculture of $A$. pullulans

and S. cerevisiae. Carbon dioxide emission rate (upper) and total carbon dioxide emission (lower) during 6-d alcoholic fermentation in YNB medium containing 10\% (w/v) glucose (a) or in a mixture of an equal weight of YNB medium and intact grape berries (b). Data represent mean values and standard deviations from three independent experiments. $A p$, inoculated with $A$. pullulans (red); $S c$, inoculated with $S$. cerevisiae (pink); $A p+S c$, coinoculated with A. pullulans and S. cerevisiae (violet). Asterisks indicate statistically significant increases in carbon dioxide emission compared with $S c$ ( $t$-test, $p<0.05)$.

Fig. 5 Assimilation of possible carbon sources in grape berries by A.pullulans and $S$. cerevisiae. Graphs indicate growth curves of A. pullulans (Ap, red) and S. cerevisiae (Sc, pink) in YNB medium containing $0.5 \%$ glucose (a), sucrose (b), CMC (c), cellobiose (Cel, d), polygalacturonic acid (polyGalUA, e), galacturonic acid (GalUA, f), $\omega$ hydroxypalmitic acid (C16:0- $\omega \mathrm{OH}, \mathbf{g})$, palmitic acid (C16:0, h), $\omega$-carboxypalmitic acid $(\mathrm{C} 16: 0-\omega \mathrm{COOH}, \mathbf{i})$, or no carbon source $(\mathbf{j})$. Data represent mean values and standard deviations from three independent experiments. Asterisks indicate statistically significant increases in carbon dioxide emission compared with $S c$ ( $t$-test, $p<0.05)$.

\section{Fig. 6 Assimilation of $\omega$-hydroxypalmitic acid by grape-skin residents and $S$.} cerevisiae. The graph indicates $\mathrm{OD}_{600}$ values after 24-h incubation in YNB medium containing $0.5 \% \omega$-hydroxypalmitic acid. A red dashed line shows the initial $\mathrm{OD}_{600}$ value $\left(\mathrm{OD}_{600}=0.1\right)$. Data represent mean values and standard deviations from three independent experiments. Ap, A. pullulans; Pl, P. laurentii; Sp, S. pararoseus; Rm, R. mucilaginosa; Hu, H. uvarum; Td, T. delbrueckii; Mc, M. caribbica; Dh, D. hansenii; Pt, 
P. terricola; Sc, S. cerevisiae.

2

3 Fig. 7 Cutinase-like activity of the culture supernatants of grape-skin residents and

4 S. cerevisiae. a PCL-plate clearing assay. b $p$ NPB and $p$ NPP hydrolysis assay. The 5 supernatants were obtained from fully grown 5-d cultures in a YNB medium containing $62 \%(\mathrm{w} / \mathrm{v})$ glucose. Data represent mean values and standard deviations from three

7 independent experiments. Ap, A. pullulans; $P l$, P. laurentii; Sp, S. pararoseus; Rm, $R$.

8 mucilaginosa; Sc, S. cerevisiae. Asterisks indicate statistically higher specific activity $9 \quad$ than $S c$ ( $t$-test, $p<0.05)$.

11 Fig. 8 Cutinase-like activity of recombinant ApCut1. a A phylogenetic tree of $A$. pullulans cutinase-like gene products. The number corresponds to each cutinase-like gene product in A. pullulans. ApCut1-ApCut3 (magenta) belongs to the yeast cutinase family,

14 while ApCut5 to ApCut9 (cyan) belong to the mold cutinase family. Bar, 0.2 substitutions per nucleotide position. b Expression of recombinant ApCut1 in E. coli. Left and right panels indicate CBB-stained gel and His-detect-stained gel for specific detection of Histagged proteins, respectively. $\mathbf{c}$ PCL-plate clearing assay. Bar, $1 \mathrm{~cm}$. d $p$ NPB hydrolysis assay. Data represent mean values and standard deviations from three independent experiments. An asterisk indicates a statistically higher specific activity than the negative control (IPTG (-), $t$-test, $p<0.05)$. EV, empty vector. 
Fig. 1

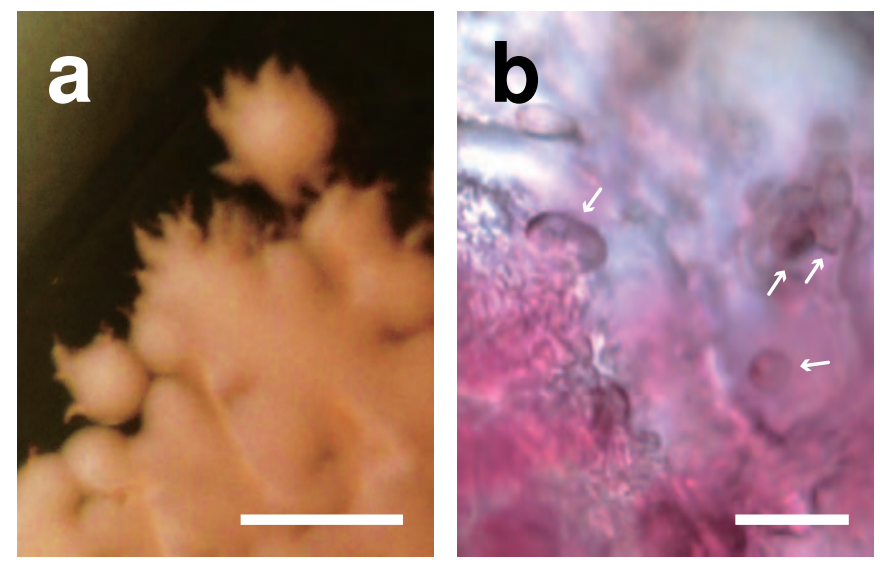


Fig. 2

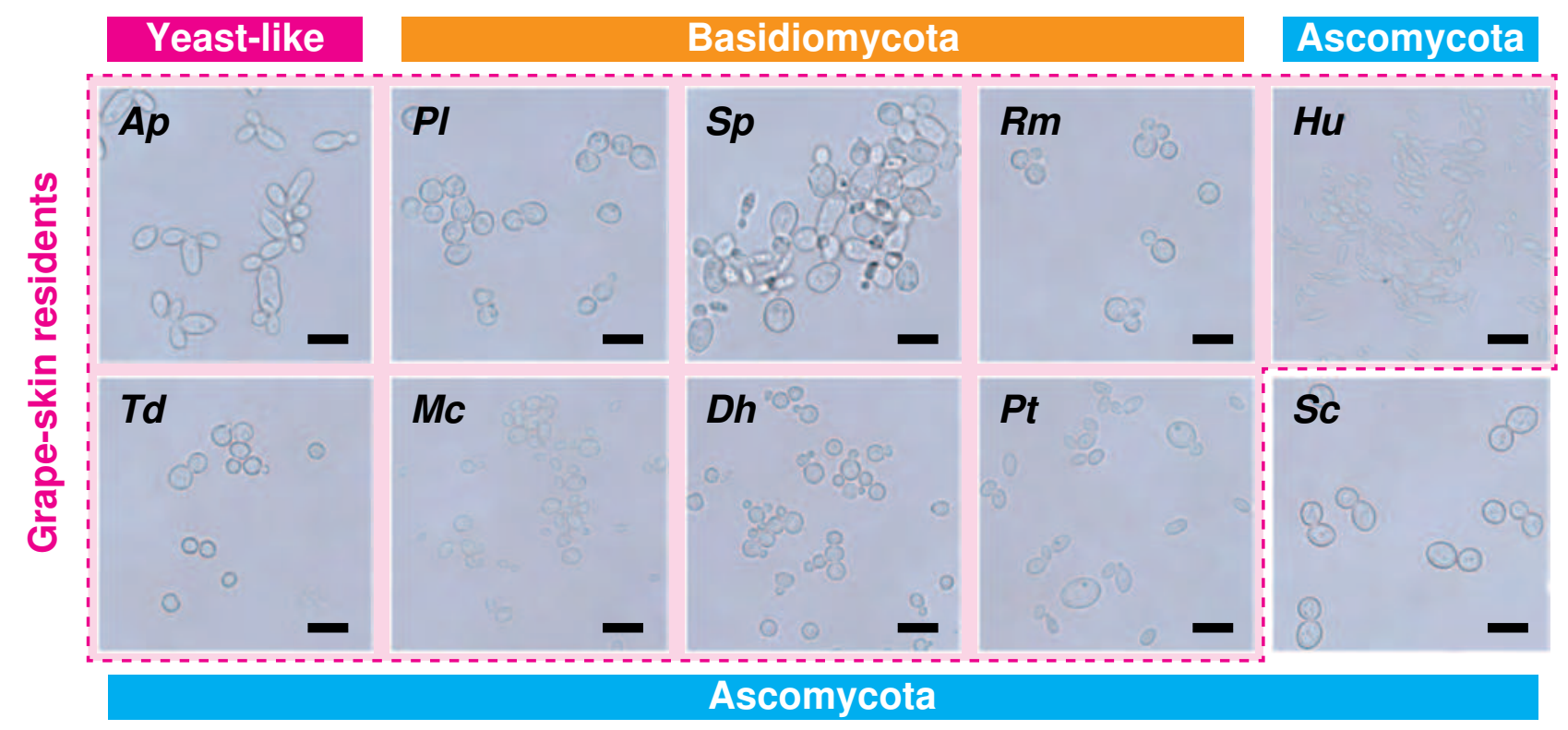


Fig. 3

a
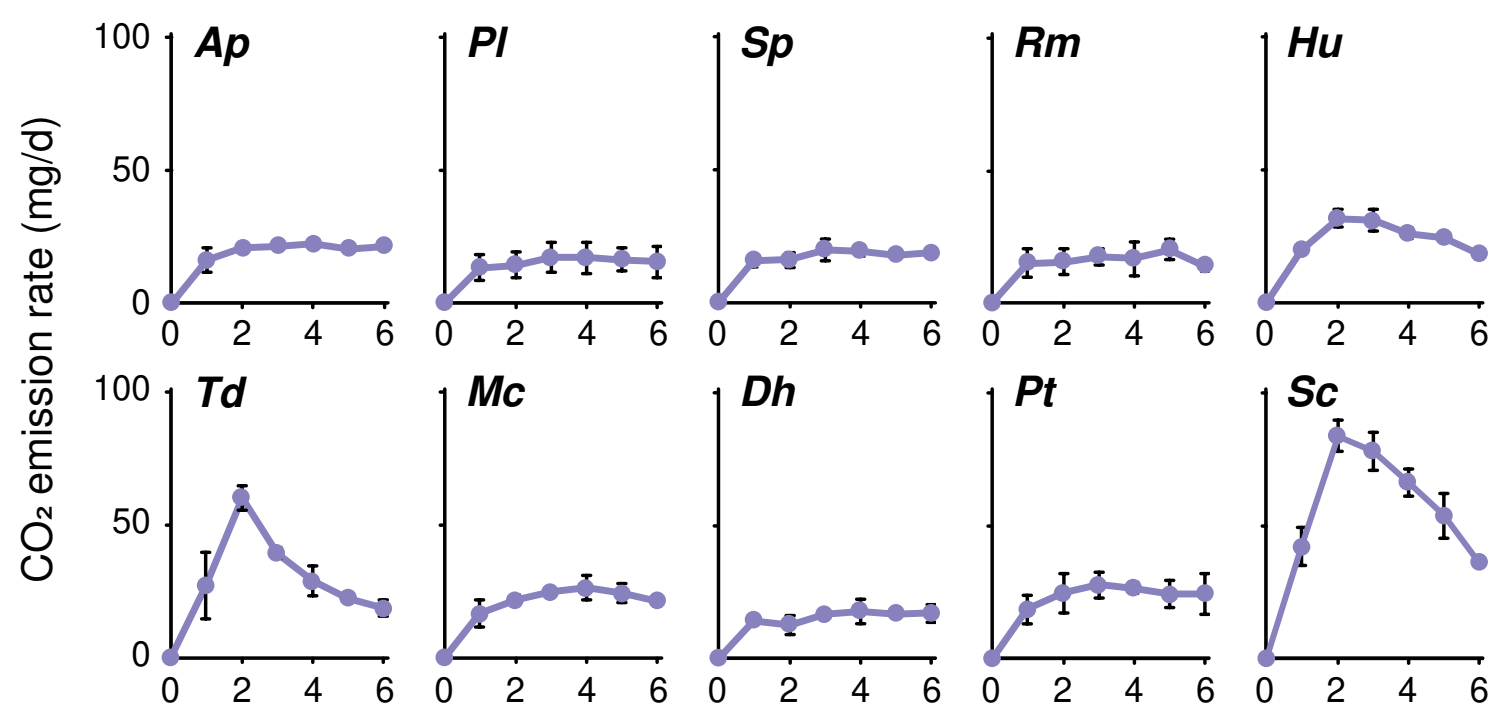

b

Time (d)

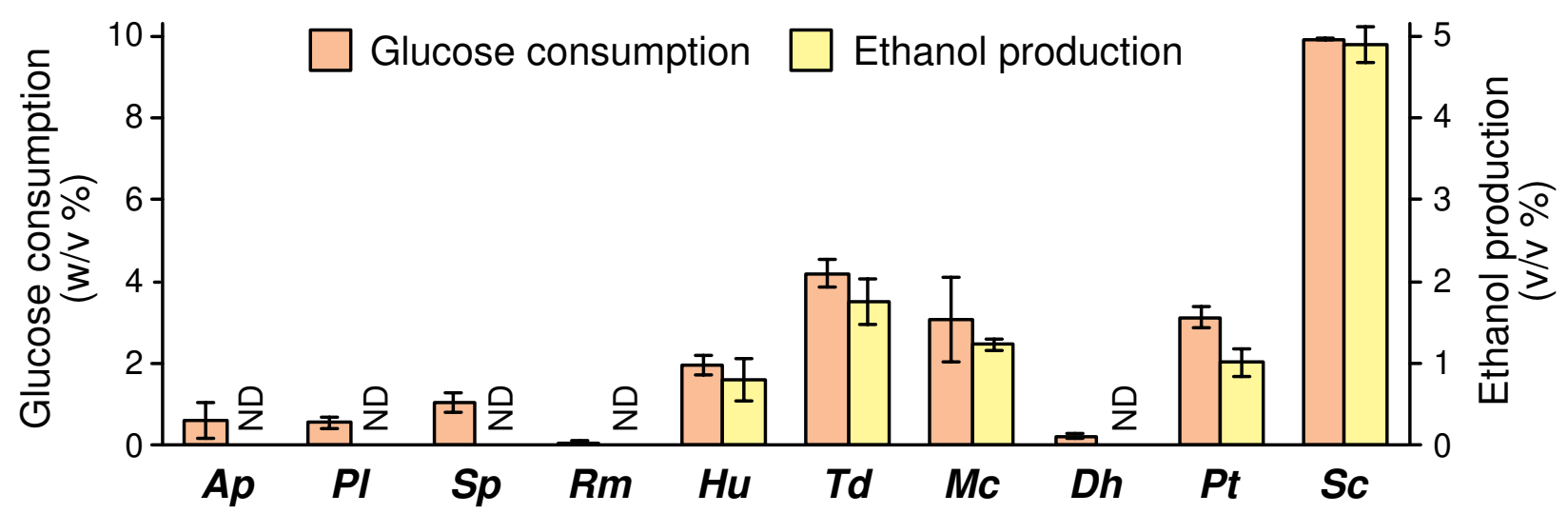


Fig. 4

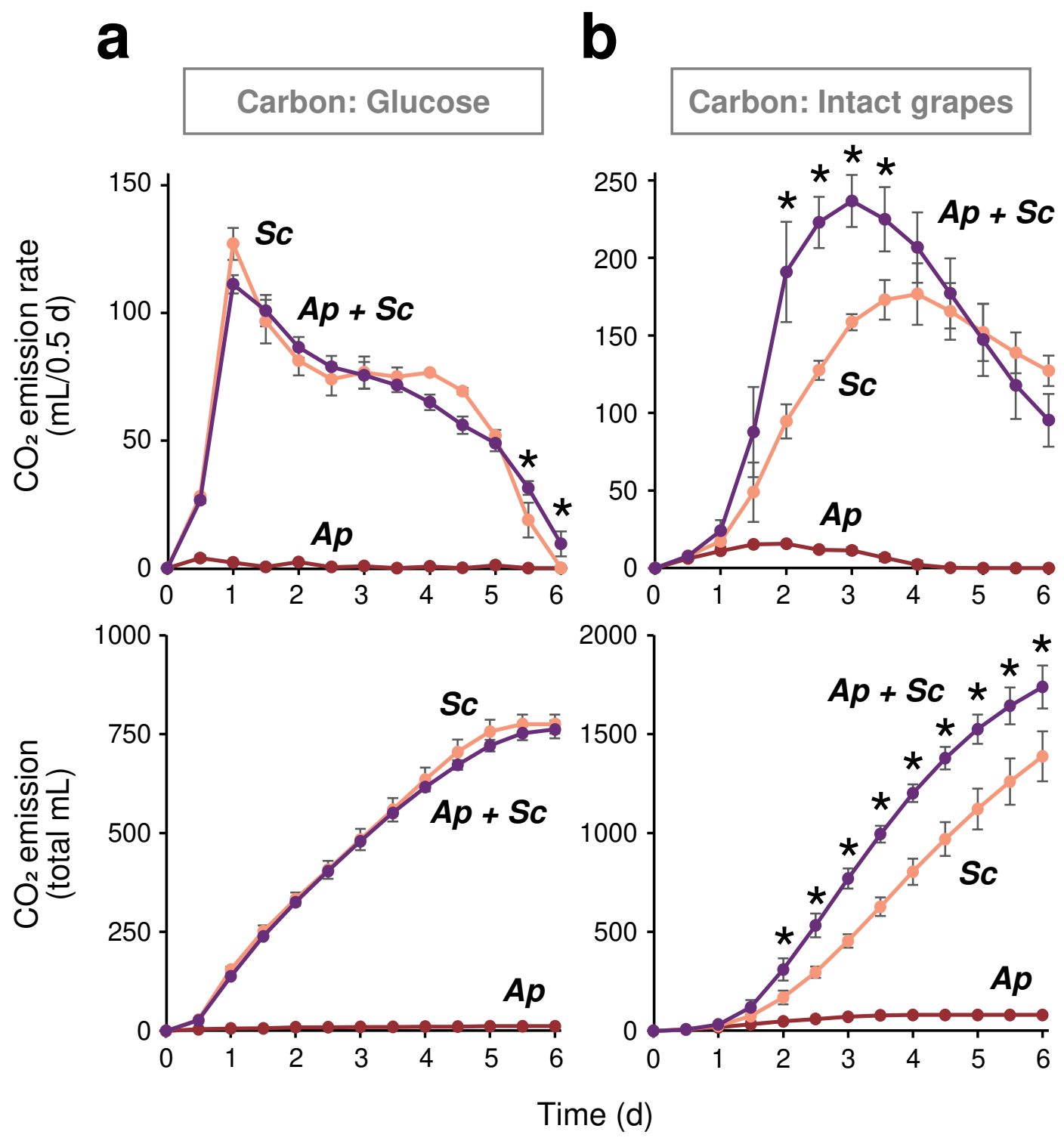


Fig. 5

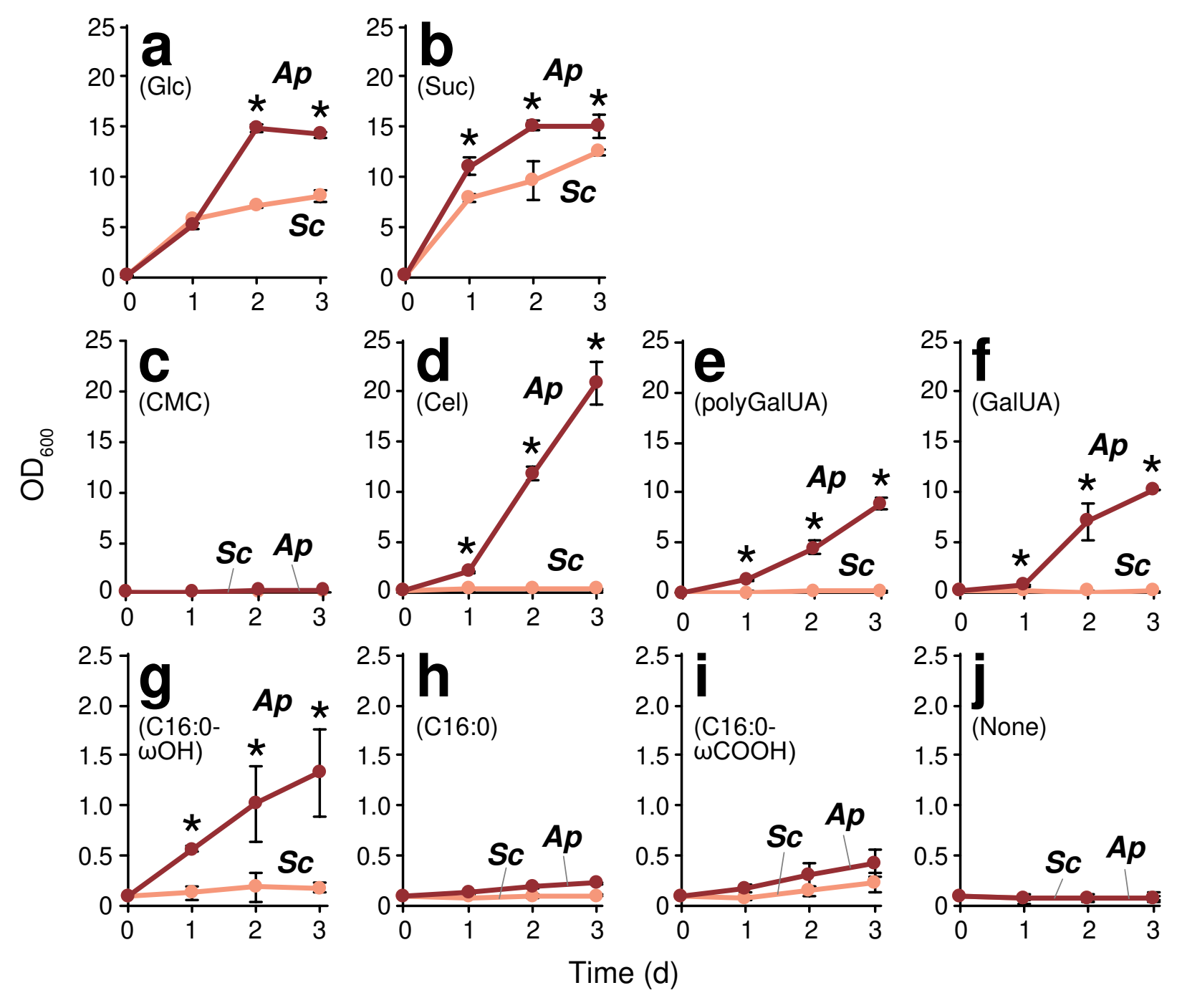


Fig. 6

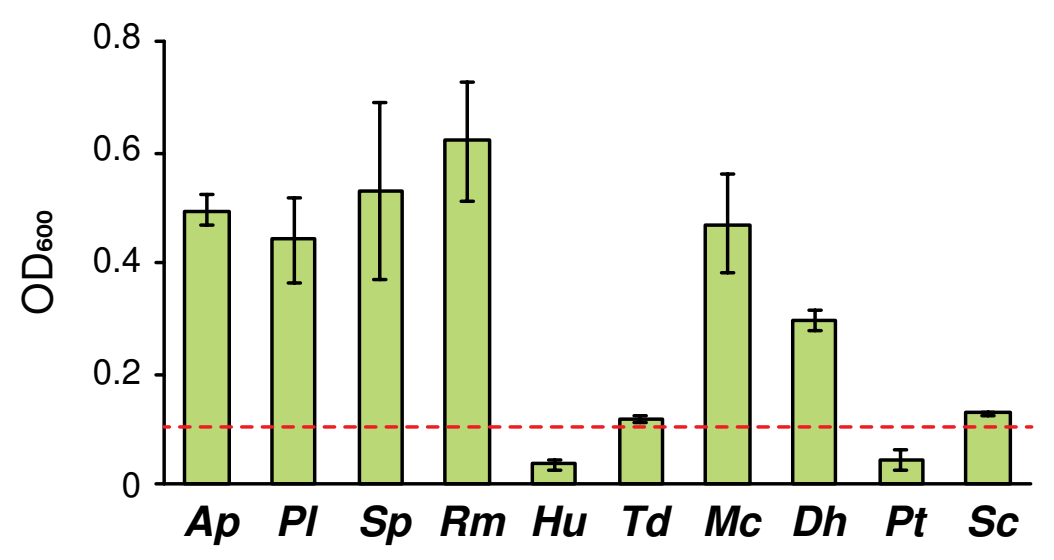


Fig. 7

a

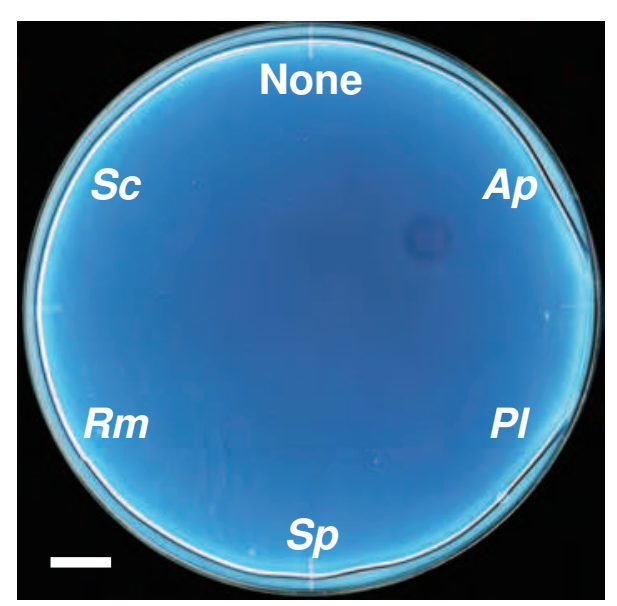

b

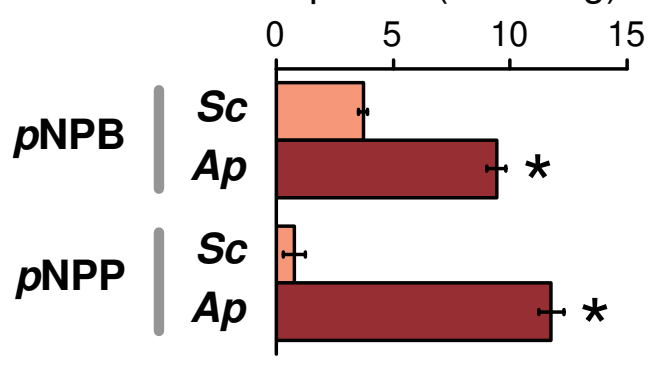


Fig. 8

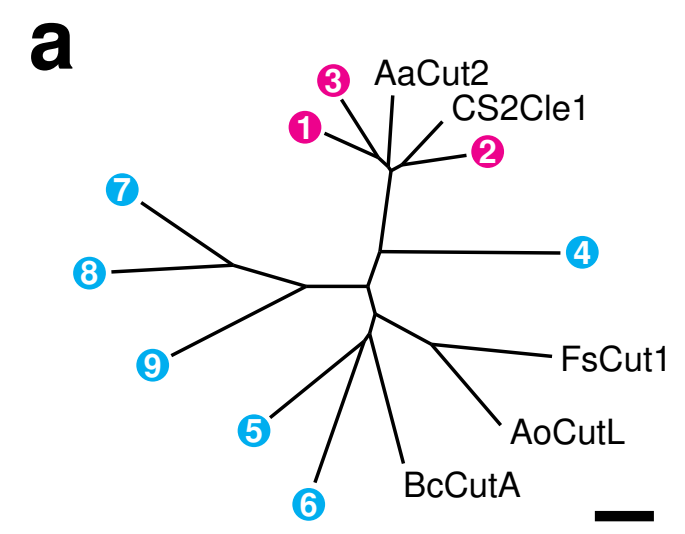

C
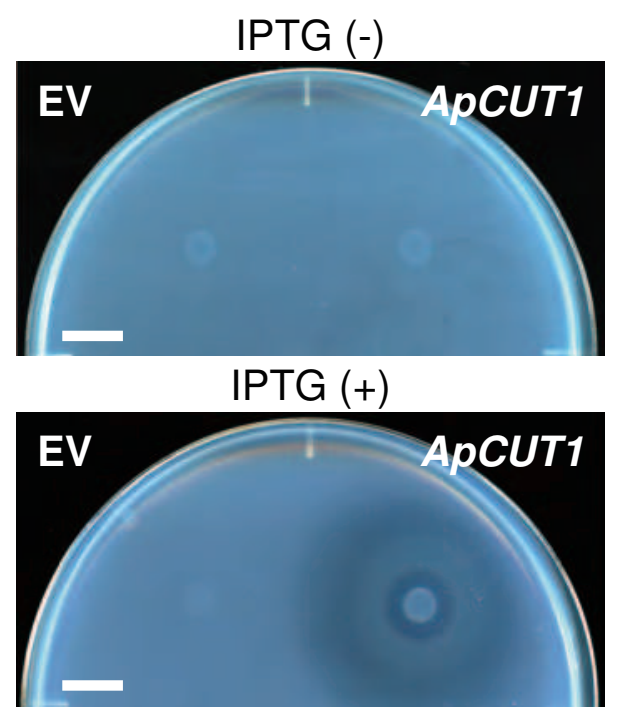

b
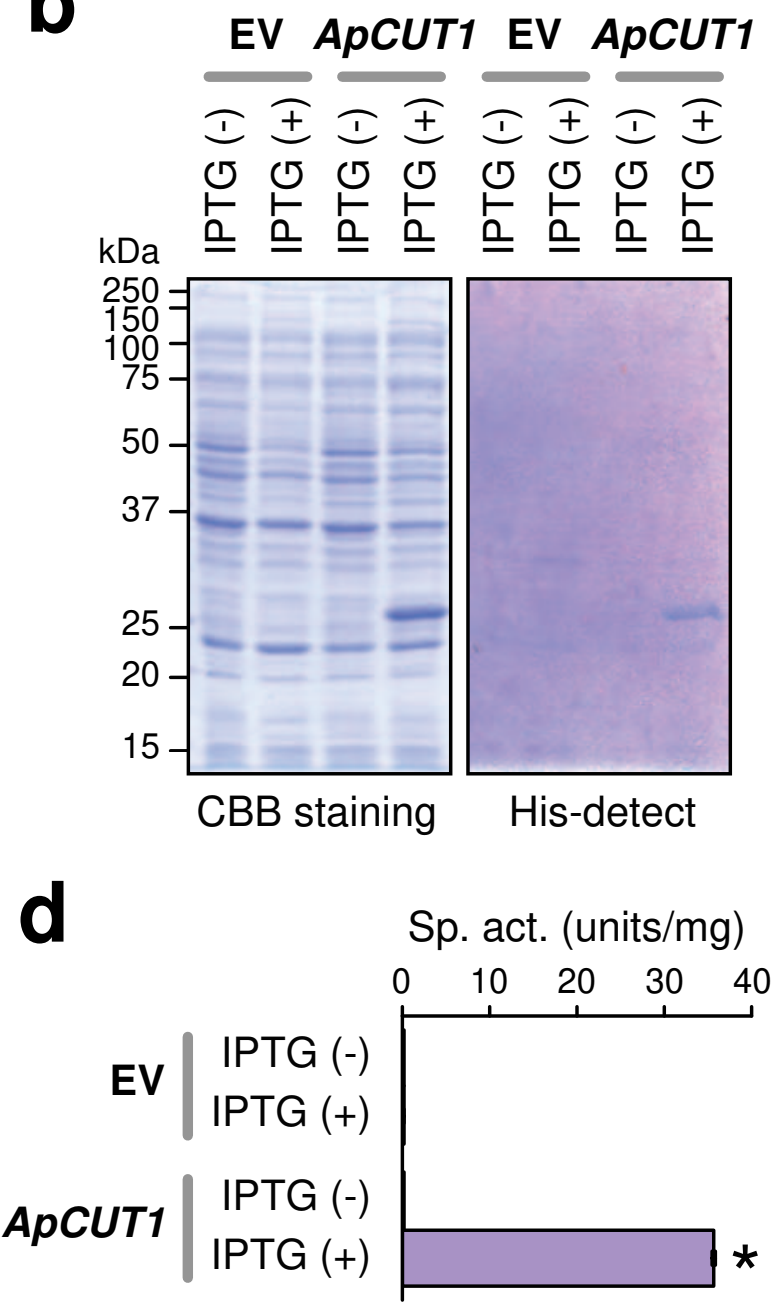


\section{Supplementary Files}

This is a list of supplementary files associated with this preprint. Click to download.

- SupplementaryData.pdf 